\title{
HD15082b, a short-period planet orbiting an A-star
}

\author{
E.W. Guenther ${ }^{1}$, A.C. Cameron ${ }^{2}$, B. Smalley ${ }^{3}$, F. Rodler ${ }^{4}$, N. Lodieu ${ }^{4}$, \\ M. Endl ${ }^{5}, \&$ K. Reif ${ }^{5}$
}

1 Thüringer Landessternwarte Tautenburg, D07778 Tautenburg, Germany [guenther@tls-tautenburg.de]

2 SUPA, School of Physics and Astronomy, University of St. Andrews, North Haugh, St. Andrews, Five KY16 9SS, UK

${ }^{3}$ Astrophysics Group, Keele University, Staffordshire ST5 5BG, UK

${ }^{4}$ Instituto de Astrofísica de Canarias, C/Vía Láctea, s/n, E38205 - La Laguna (Tenerife), Spain

${ }^{5}$ McDonald Observatory, University of Texas, Austin, TX 78712, USA

${ }^{6}$ Argelander-Institut fr Astronomie der Universität Bonn, Auf dem Huegel 71, D53121 Bonn, Germany

\begin{abstract}
Most of the known transiting extrasolar planets orbit slowly rotating F, G or K stars. In here we report on the detection of a transiting planet orbiting the bright, rapidly rotating A5 star HD15082, recently made by SuperWASP. Time resolved spectroscopic observations taken during transit show a hump caused by the planet crossing the line profile. From the analysis of the spectra, we derive the radius of the planet and find that it is orbiting retrograde in respect to the spin of the star. Because of its small distance from an A5 star, this planet must be one of the hottest planets known, which makes it relatively easy to detect it in the IR. We thus tried to detect it using the TNG but did not succeed. Using direct imaging, we search for possible companions, and found one candidate.
\end{abstract}

\section{The importance of studying close-in planets of A-stars}

Studies of transiting extra-solar planets are of key importance for understanding the nature of planets outside our Solar System, because they allow to derive their mass, diameter and their density. The more than 100 transiting planets discovered up to now thus give us a wealth of information about the structure and evolution of extrasolar planets.

However, almost all known transiting planets orbit F, G or K stars. Thus, only very little is known about the planets of A-stars. Studying such planet would however be very interesting, because planets of A-stars are exposed to a very high flux by the host star in the optical regime. By studying close-in planets of A-stars thus helps us to better understand the effects caused by the radiation of the host stars on the planets. However, important for the evaporation of the atmosphere of a planet is the amount of radiation which the planet receives shortward of the Ly- $\alpha$ line, not so much the radiation in the optical regime. Whether planets of A-stars also receive more photons in this wavelength regime is less obvious. In the classical view, A-stars do not have an outer convection zone and thus do not have a chromosphere, or a corona. 
Although there is now growing evidence that this classical view is not be strictly true, the chromospheres and coronae of A-stars are certainly not like those of later type stars (Simon et al. 2002; Hempel et al. 2005; Schröder \& Schmitt 2007). Anyhow, in order to better understand the interaction between close-in planets and their host stars, it is important to study planets of A-stars, because A-stars are quite different from G-stars.

In here we report on new observations obtained for the close-in, transiting planet orbiting the A5 star HD15082 (WASP-33). The discovery of HD15082b was presented by Cameron et al. (2010). Because the detection and the properties of the planet were already described in detail in this article, we will just briefly summarize the previous results in here, and focus on new ones.

\section{A unique star/planet system}

The light curve of HD15082b (WASP-33b) obtained by SuperWASP shows flatbottomed, planet-like transits recurring every $1.22 \mathrm{~d}$. We obtained three time-series of spectra during transit with echelle spectrographs. The spectra were taken with the 2-m-Alfred Jensch telescope at Tautenburg observatory, the 2.7-m Harlan J. Smith Telescope at McDonald Observatory, and the Nordic-Optical-Telescope (NOT) at the Observatorio del Roque de los Muchachos. These time-series of spectra clearly show the characteristic signature of a transiting planet. The analysis of the spectra not only allowed us to confirm that there is transiting planet but it was also found that this planet orbits retrograde.

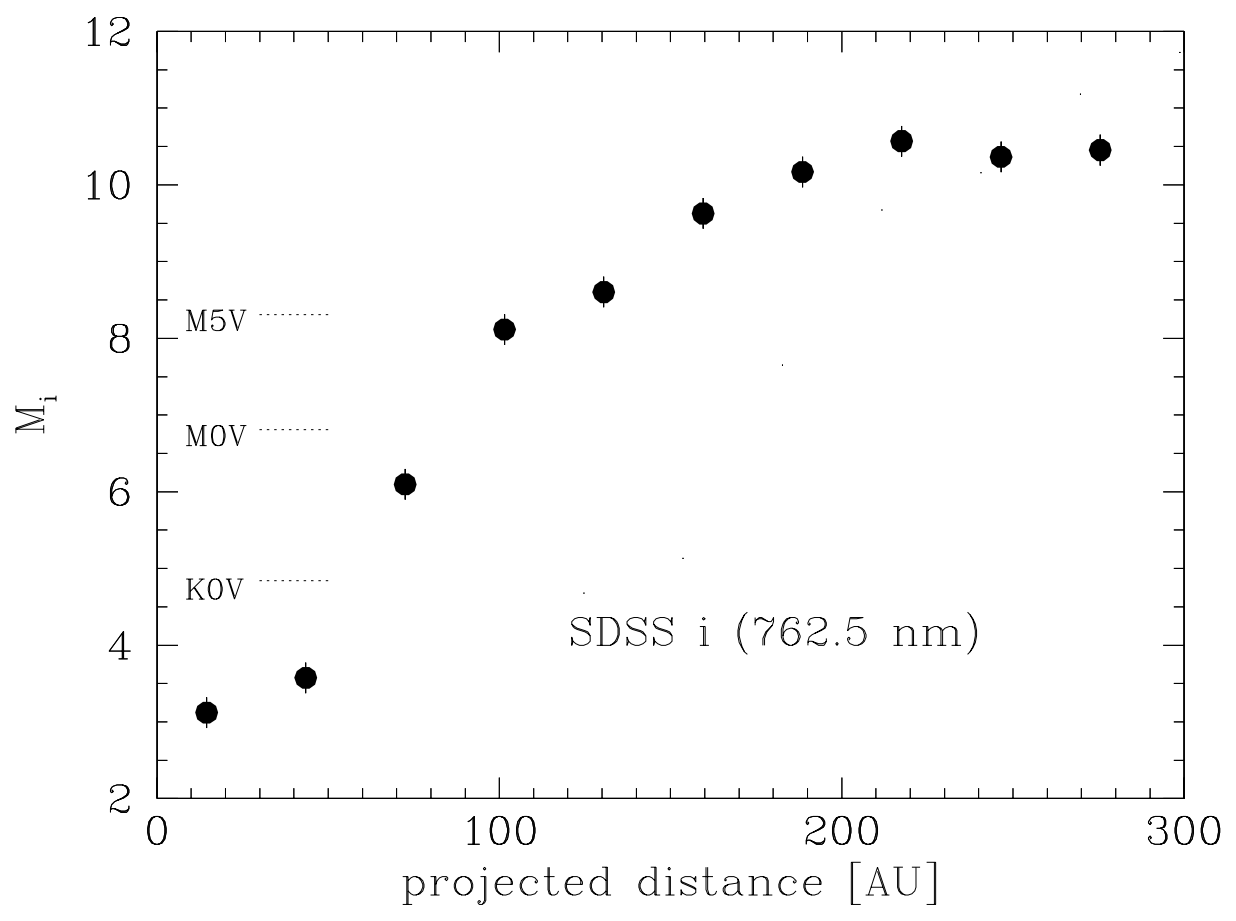

Figure 1: Upper limit for possible companions close to HD15082. 
We additionally obtained 29 radial-velocity measurements with the Alfred Jensch telescope in order to constrain the mass of the planet. Although an iodine cell was used for the observations, the huge v sin i of the star of $90 \mathrm{~km} \mathrm{~s}^{-1}$ limited the accuracy of our measurements to about $700 \mathrm{~m} \mathrm{~s}^{-1}$. We derive an upper limit for the mass of the planet of $4.1 M_{\mathrm{Jup}}$. Combined with the radius of $1.497 \pm 0.045 R_{\mathrm{Jup}}$, this gives us an upper limit for the density of $1.2 \mathrm{~g} \mathrm{~cm}^{-3}$. The planet thus may well belong to the class of low-density planets, which would mean that the evaporation rate could be high.

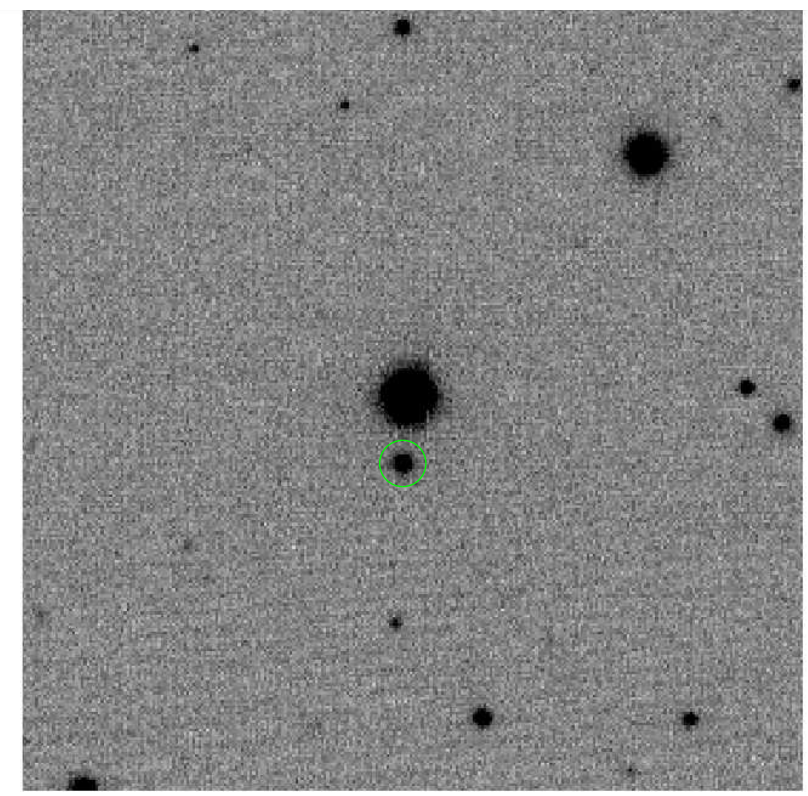

$20 \quad 40$

120

140

160

180

200

Figure 2: B-band image taken with the 30-cm-astrograph at Hoher List observatory (10x10 arcmin, north is up and east is left). HD15082 is the bright star in the middle, the companion candidate is marked with a circle.

It thus turns out that the planet has unique properties. The orbital separation is only $0.02555 \pm 0.00017 \mathrm{AU}$, or $3.83 \pm 0.09 R_{\text {star }}$. Since the star has a temperature of $7430 \pm 100 \mathrm{~K}$, this planet receives about five times as much radiation in the visual regime than a planet orbiting a solar-like star at the same distance. Simon \& Landsman (1997) studied the emission of many A to F stars at $1900 \AA$, and find that the relative flux of A-stars is on average higher than that of late type stars. However, the flux at $1900 \AA$ has scatter of $\pm 0.4 \mathrm{mag}$ for stars of the same spectral type. The flux of HD15082 at $1965 \AA$ is $(1.43 \pm 0.44) \times 10^{-12} \mathrm{erg} \mathrm{s} \mathrm{cm}^{2} / \AA$ which means that this star is much brighter than the sun in this wavelength regime. More difficult is it to estimate the flux short-ward of Ly- $\alpha(\lambda=121.5668 \mathrm{~nm})$ which is the essential parameter in order to estimate the mass-loss of the planet.

\section{Searching for possible companions}

Interestingly, it turned out that HD15082b (WASP-33b) orbits retrograde. One of the possibilities how the planet got into such an orbit is the interaction with a massive body. If this massive body were a star, it could easily be detected. In order to search 
for a possible companion we took images using AstraLux, the Calar Alto Lucky Imaging Camera mounted on the 2.2-m-telescope. By selecting only the best $1 \%$ of 4000 images each exposed for only $0.015360 \mathrm{~s}$, we obtained an almost diffraction limited image of HD15082 in the SDSS i' and z' filter. The field-of-view of the camera is about 8x8 arcsec, corresponding to about $900 \mathrm{AU}$ at the distance of HD15082. The results are shown in Fig.1. Our observations exclude a companion with a spectral type of M5V, or earlier at a distance from 100 to 450 AU from HD15082.

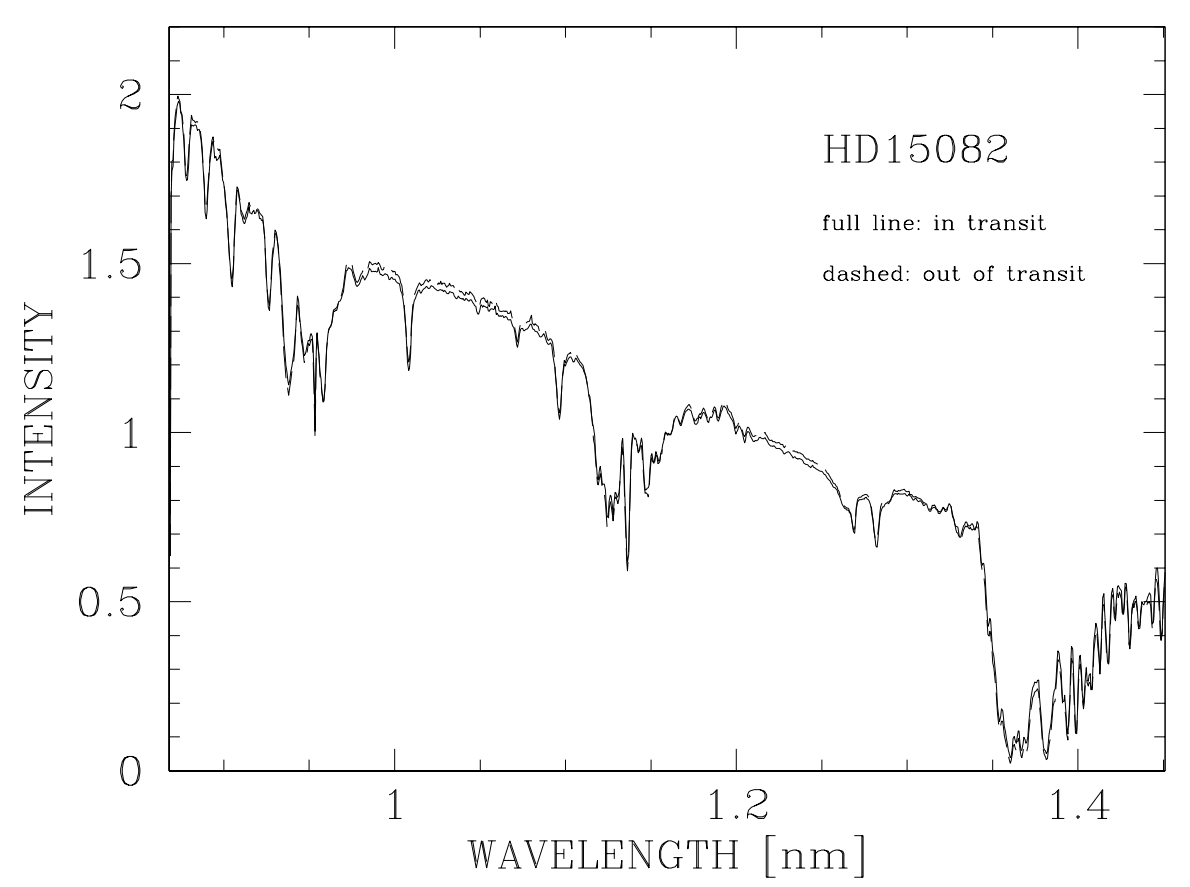

Figure 3: Spectrum of HD15082b taken in and out of the secondary transit. The planet is not detected.

There is however, a companion candidate at a distance of 44 arcsec, GSC0283101138 (2MASS02265167+3732133) (Fig.2). This star has $m_{B}=12.22 \pm 0.19$, and $m_{V}=11.28 \pm 0.09$ (Zacharias et al. 2005). If this star is a true companion, it would be at a projected distance of $5100 \mathrm{AU}$, and it would be $M v=5.96 \pm 0.29$, corresponding to a K0V star. Using the data given in SIMBAD, we find $B-V=0.94 \pm 0.21$ which corresponds to a $\mathrm{K} 2 \mathrm{~V}$ star, and $V-K=1.88 \pm 0.09$ which corresponds to a G9V star. The photometric distance of GSC2831-01138 thus is consistent with a companion. However, the proper motion of HD15082 and GSC2831-01138 are somewhat different (Tab.1).

\section{Spectroscopic observations of the secondary transit}

Because the planet is so close to a hot A-type star, at least the side facing the star must be very hot. A simple model leads to a temperature of about $2800 \mathrm{~K}$ but the real temperature could even be higher. During the secondary transit of an extrasolar 
Detection and Dynamics of Transiting Exoplanets

\begin{tabular}{|c|c|c|c|}
\hline star & $\begin{array}{l}\text { pmRA } \\
{[\mathrm{mas} / \mathrm{yr}]}\end{array}$ & $\begin{array}{l}\text { pmDEC } \\
\text { [mas/yr] }\end{array}$ & source \\
\hline \multirow[t]{4}{*}{ WASP33b } & $-0.31 \pm 1.05$ & $-8.82 \pm 0.83$ & 1 \\
\hline & $-0.3 \pm 1.0$ & $-8.8 \pm 0.8$ & 2 \\
\hline & $-1.0 \pm 0.7$ & $-8.5 \pm 1.0$ & 3 \\
\hline & $-0.04 \pm 1.7$ & $-8.66 \pm 1.6$ & 4 \\
\hline \multirow[t]{4}{*}{ GSC283101138 } & $-7.5 \pm 4.0$ & $-8.5 \pm 3.8$ & 1 \\
\hline & $-3.4 \pm 1.8$ & $-5.5 \pm 0.7$ & 2 \\
\hline & $-1.6 \pm 2.4$ & $-4.5 \pm 0.9$ & 3 \\
\hline & $-10.2 \pm 2.1$ & $-4.6 \pm 2.2$ & 4 \\
\hline
\end{tabular}

1 The Hipparcos and Tycho Catalogues (Perryman et al. 1997)

2 NOMAD Catalog (Zacharias et al. 2005)

3 UCAC3 Catalog (Zacharias et al. 2010)

4 PPMX Catalog (Röser et al 2008)

planet, the star occults the planet. Shortly before that moment, the planet is observed at its maximum brightness. Thus, by observing the planet before, during and after the secondary transit, it is possible to determine the amount of radiation coming from the planet alone. Such secondary eclipses have been observed most notably from space but have also been detected from the ground.

Up to now, the temperatures of the planets that have been studied are typically about 1000 K (GJ436b: 712 $\pm 36 \mathrm{~K}$, Deming et al. 2007; TrES-1: $1060 \pm 50 \mathrm{~K}$, Charbonneau et al. 2005; HD189733b: $1117 \pm 42$ K, Deming et al. 2006; HD209458b: $1130 \pm 150$ K, Deming et al. 2005; HD189733: $973 \pm 33 \mathrm{~K}$, Knutson et al. 2007). It would thus be interesting to study the properties of a planet with a temperature of $2800 \mathrm{~K}$.

The planet should be $m_{J}=15.11, m_{H}=14.8, m_{K}=14.4$, or $\approx 7$ mag fainter than the star. The problem thus is not the brightness of the planet itself but the brightness difference between the star and the planet. Using the Telescopio Nationale Galileo (TNG) at the Observatorio del Roque de los Muchachos and its NICS spectrograph, we have obtained spectra in and out of transit. We used the IJ-grism which covers the wavelength range from 0.9 to $1.45 \mu \mathrm{m}$. With a slit-width of 1 arcsec, NICS achieves a resolution of $\lambda / \Delta \lambda=500$. In order to minimize the flux-losses, we opened the slit as far as possible, which is 2 arcsec. The object was observed in-transit for about half an hour and out-of transit for one hour. Unfortunately, the night was partly cloudy which limited the time for which we could observe the object.

The spectra are shown in Fig.3. Unfortunately, due to clouds, we can only derive an upper limit of $1.2 \%$ for the relative flux of the planet. We expect that the flux of the planet is $\geq 0.1 \%$ to $\geq 0.2 \%$ in the NIR. This is clearly insufficient for the detection of the planet. Spectra of higher signal-to-noise ratio or photometric data of sufficient quality are needed in order to detect the secondary eclipse of the planet.

Acknowledgements. We are grateful to the user support group of TNG for all their help and assistance for preparing and carrying out the observations. 


\section{EPJ Web of Conferences}

\section{References}

Cameron, A.C., et al. 2010, MNRAS, 407, 507

Charbonneau , D., et al. 2005, ApJ, 626, 523

Deming, D., Seager, S., Richardson, L. J., \& Harrington, J. 2005, Nature, 434, 740

Deming, D., Harrington, J., Seager, S., \& Richardson, L.J. 2006 ApJ, 644, 560

Deming, D., Harrington, J., Laughlin, G., Seager, S., Navarro, S.B., Bowman, W.C., \& Horning, K. 2007, ApJL, 667, L199

Hempel, M., Robrade, J., Ness, J.-U., \& Schmitt, J.H.M.M. 2005, A\&A, 440, 727

Knutson , H.A., et al. 2007, Nature, 447, 183

Perryman, M.A.C., and ESA 1997, The Hipparcos and Tycho catalogues. Astrometric and photometric star catalogues derived from the ESA Hipparcos Space Astrometry Mission, Publisher: Noordwijk, Netherlands: ESA Publications Division, 1997, Series: ESA SP Series vol no: 1200

Röser, S., Schilbach, E., Schwan, H., Kharchenko, N. V., Piskunov, A. E., \& Scholz, R.-D. 2008, A\&A, 488, 401

Schröder, C., \& Schmitt, J.H.M.M. 2007 A\&A, 475, 677

Simon, T., \& Landsman, W.B. 1997, ApJ, 484, 360

Simon, T., Ayres, T.R., Redfield, S., \& Linsky, J.L. 2002, ApJ, 579, 800

Zacharias, N., Monet, D. G., Levine, S. E., Urban, S. E., Gaume, R., \& Wycoff, G. L. 2005, VizieR Online Data Catalog, 1297, 0

Zacharias, N., et al. 2010, AJ, 139, 2184 